\title{
G-H-KKM SELECTIONS WITH APPLICATIONS TO MINIMAX THEOREMS
}

\author{
RAM U. VERMA \\ International Publications \\ Division of Mathematical Sciences \\ 12046 Coed Drive \\ Orlando, FL 32826 USA
}

(Received September, 1998; Revised November, 1999)

Based on the G-H-KKM selections, some nonempty intersection theorems and their applications to minimax inequalities are presented.

Key words: G-H-KKM Selections, Intersection Theorems, Minimax Theorems.

AMS subject classifications: $49 \mathrm{~J} 40$.

\section{Introduction}

Minimax inequalities have numerous applications to variational inequalities, while variational inequalities turn out to be a powerful tool to the solvability of problems in elasticity and plasticity theory, heat conduction, diffusion theory, optimization theory, mathematical economics, and others.

Chang and Zhang [1] introduced the notion of the generalized quasiconcavity and obtained some nonempty intersection theorems and their applications to minimax inequalities in a linear topological space setting. Recently, Tan [4] extended this notion to the case of a G-convex space with applications to minimax theorems and saddle points. Our aim here is to present some G-H-KKM selection theorems and related applications to minimax inequalities in a $\mathrm{G}-\mathrm{H}$-space setting.

Let $X$ be a topological space, $P(X)$ denote the power set of $X$, and $\langle X\rangle$, a family of all nonempty finite subsets of $X$. Let $\Delta^{n}$ denote a standard $(n-1)$ simplex $\left\{e_{1}, e_{2}, \ldots, e_{n}\right\}$ of $R^{n}$.

Definition 1.1: A triple $(X, H,\{p\})$ is called a $G-H$-space [6] if $X$ is a topological space and $H:\langle X) \rightarrow P(X) \backslash\{\emptyset\}$ is a mapping such that:

(i) For each $F, G \in\langle X\rangle$, there exists $F_{1} \subset F$ such that $F_{1} \subset G$ implies $H\left(F_{1}\right) \subset H(G)$.

(ii) For $F=\left\{x_{1}, x_{2}, \ldots, x_{n}\right\} \in\langle X\rangle$, there is a continuous mapping $p: \Delta^{n} \rightarrow H(F)$ such that for $\{i 1, i 2, \ldots, i k\} \subset\{1,2, \ldots, n\}$, we have $p\left(\left\{e_{i 1}, e_{i 2}, \ldots, e_{i k}\right\}\right) \subset$ $H\left(\left\{x_{i 1}, x_{i 2}, \ldots, x_{i k}\right\}\right)$, where $\left\{x_{i 1}, x_{i 2}, \ldots, x_{i k}\right] \subset F$. 
A subset $D$ of $X$ is called finitely $G$-H-closed in $X$ if for each $A \in\langle X\rangle$, there exists $\underset{\sim}{A} \subset A$ such that $D\left({ }^{-}\right) H(\underset{\sim}{A})$ is closed in $H(\underset{\sim}{A})$.

A subset $K$ of $X$ is said to be compactly closed in $X$ if $K\left(^{-}\right) L$ is closed in $L$ for all compact subsets $L$ of $X$.

Definition 1.2: Let $(X, H,\{p\})$ be a G-H-space and $T: X \rightarrow P(X)$ a multivalued mapping. $T$ is called a $G-H-K K M$ mapping if for each $\left\{x_{1}, x_{2}, \ldots, x_{n}\right\} \in\langle X\rangle$, there exists $\left\{x_{i 1}, x_{i 2}, \ldots, x_{i k}\right\} \subset\left\{x_{1}, x_{2}, \ldots, x_{n}\right\}$ such that

$$
H\left(\left\{x_{i 1}, x_{i 2}, \ldots, x_{i k}\right\}\right) \subset\left(\frac{k}{j=1}\right) T\left(x_{i j}\right) \text { for }\{i 1, i 2, \ldots, i k\} \subset\{1,2, \ldots, n\} .
$$

Definition 1.3: Let $(X, H,\{p\})$ be a G-H-space and let $M_{1}, M_{2}, \ldots, M_{n}$ be subsets of $X$. A subset $\left\{x_{1}, x_{2}, \ldots, x_{n}\right\} \in\langle X\rangle$ is said to be a $G-H-K K M$ selection for $M_{1}, M_{2}, \ldots, M_{n}$ if for any $\left\{x_{i 1}, x_{i 2}, \ldots, x_{i k}\right\} \subset\left\{x_{1}, x_{2}, \ldots, x_{n}\right\}$, we have

$$
H\left(\left\{x_{i 1}, x_{i 2}, \ldots, x_{i k}\right\}\right) \subset\left(\frac{k}{j=1}\right) M_{i j} \text { for }\{i 1, i 2, \ldots, i k\} \subset\{1,2, \ldots, n\} .
$$

This generalizes the notion of a KKM selection in a pseudoconvex space by Joo' and Kassay [2].

Definition 1.4: Let $X$ be nonempty set and $(Y, H,\{p\})$ a G-H-space. Let $f: X \times Y \rightarrow R, e: Y \rightarrow R$ and $h: X \rightarrow R$ be functions. The function $f$ is said to be 0 generalized G-H-quasiconcave (resp. 0-generalized G-H-quasiconvex) in its first variable $x$ if for each $\left\{x_{1}, x_{2}, \ldots, x_{n}\right\} \in\langle X\rangle$, there exists $\left\{v_{1}, v_{2}, \ldots, v_{n}\right\} \in\langle Y\rangle$ such that for each $\left\{v_{i 1}, v_{12}, \ldots, v_{i k}\right\} \subset\left\{v_{1}, v_{2}, \ldots, v_{n}\right\}$ and for any $y_{0} \in H\left(\left\{v_{i 1}, v_{i 2}, \ldots, v_{i k}\right\}\right)$, we have

$$
\begin{gathered}
\min _{1 \leq j \leq k}\left[f\left(x_{i j}, y_{0}\right)+e\left(y_{0}\right)-h\left(x_{i j}\right)\right] \leq 0 \\
\left(\text { resp. } \max _{1 \leq j \leq k}\left[f\left(x_{i j}, y_{0}\right)+e\left(y_{0}\right)-h\left(x_{i j}\right)\right] \geq 0\right),
\end{gathered}
$$

where $\{i 1, i 2, \ldots, i k\} \subset\{1,2, \ldots, n\}$.

This generalizes the notion of a 0 -generalized quasiconcavity (0-generalized quasiconvexity) by Chang and Zhang [1].

\section{G-H-KKM Theorems and Applications}

In this section we first recall and obtain some auxiliary results and then establish some minimax theorems.

Lemma 2.1: [7] Let $(X, H,\{p\})$ be a $G$-H-space and $M_{1}, M_{2}, \ldots, M_{n}$ be finitely $G$-H-closed subsets of $X$. Suppose that $M_{1}, M_{2}, \ldots, M_{n}$ have a $G-K K M$ selection. Then $\left({ }^{-}\right)_{i=1}^{n} M_{i} \neq \emptyset$.

Proposition 2.1: Let $X$ be a nonempty set and $(Y, H,\{p\})$ a G-H-space. Let $f: X \times Y \rightarrow R, e: Y \rightarrow R$ and $h: X \rightarrow R$ be functions. Then the following statements are equivalent:

(a) A mapping $T: X \rightarrow P(Y)$ defined by

$$
T(x)=\{y \in Y: f(x, y)+e(y)-h(x) \leq 0\}
$$


$($ resp. $T(x)=\{y \in Y: f(x, y)+e(y)-h(x) \geq 0\})$,

is a $G-H-K K M$ mapping.

(b) $f$ is 0-generalized G-H-quasiconcave (resp. 0-generalized G-H-quasiconvex) in its first variable $x$.

Proof: $(a) \Rightarrow(b) \quad$ Since $T$ is G-H-KKM, it implies for each $\left\{x_{1}, x_{2}, \ldots, x_{n}\right\} \in\langle X\rangle$ and corresponding $\left\{v_{1}, v_{2}, \ldots, v_{n}\right\} \in\langle Y\rangle$ that there exist $\left\{x_{i 1}, x_{i 2}, \ldots, x_{i k}\right\} \subset\left\{x_{1}, x_{2}, \ldots\right.$, $\left.x_{n}\right\},\left\{v_{i 1}, v_{i 2}, \ldots, v_{i k}\right\} \subset\left\{v_{1}, v_{2}, \ldots, v_{k}\right\}$ and any $y_{0} \in H\left(\left\{v_{i 1}, v_{i 2}, \ldots, v_{i k}\right\}\right)$ such that

$$
H\left(\left\{v_{i 1}, v_{i 2}, \ldots, v_{i k}\right\}\right) \subset\left(\frac{k}{j=1}\right) T\left(x_{i j}\right) .
$$

This implies $y_{0} \in\left(\frac{k}{j=1}\right) T\left(x_{i j}\right)$, and as a result, there exists some index $m(i \leq m \leq k)$ such that $y_{0} \in T\left(x_{i m}\right)$. Hence, $f\left(x_{i m}, y_{0}\right)+e\left(y_{0}\right)-h\left(x_{i m}\right) \leq 0$ (resp. $f\left(x_{i m}, y_{0}\right)+$ $\left.e\left(y_{0}\right)-h\left(x_{i m}\right) \geq 0\right)$. It follows that

$$
\begin{gathered}
\min _{1 \leq j \leq k}\left[f\left(x_{i j}, y_{0}\right)+e\left(y_{0}\right)-h\left(x_{i j}\right)\right] \leq 0 \\
\text { (resp. } \left.\max _{1 \leq j \leq k}\left[f\left(x_{i j}, y_{0}\right)+e\left(y_{0}\right)-h\left(x_{i j}\right)\right] \geq 0\right) .
\end{gathered}
$$

$(b) \Rightarrow(a) \quad$ Since $f$ is 0 -generalized G-H-quasiconcave (resp. 0-generalized G-Hquasiconvex) in $x$, it implies for any $\left\{x_{1}, x_{2}, \ldots, x_{n}\right\} \in\langle X\rangle$ and $\left\{v_{1}, v_{2}, \ldots, v_{n}\right\} \in\langle Y\rangle$, there exist $\left\{v_{i 1}, v_{i 2}, \ldots, v_{i k}\right\} \subset\left\{v_{1}, v_{2}, \ldots, v_{n}\right\}$, and any $y_{0} \in H\left(\left\{v_{i 1}, v_{i 2}, \ldots, v_{i k}\right\}\right)$ such that

$$
\begin{gathered}
\min _{1 \leq j \leq k}\left[f\left(x_{i j}, y_{0}\right)+e\left(y_{0}\right)-h\left(x_{i j}\right)\right] \leq 0 \\
\left(\text { resp. } \max _{1 \leq j \leq k}\left[f\left(x_{i j}, y_{0}\right)+e\left(y_{0}\right)-h(x) \geq 0\right) .\right.
\end{gathered}
$$
It follows that there exists some index $m(1 \leq m \leq k)$ such that $y_{0} \in T\left(x_{m}\right) \subset\left(\frac{k}{j=1}\right)$
$T\left(x_{i j}\right)$. This completes the proof.

Theorem 2.1: Let $X$ be a nonempty set and $(Y, H,\{p\})$ a G-H-space such that $H(F)$ is compact for all $F \in\langle Y\rangle$. Suppose that $f: X \times Y \rightarrow R, e: Y \rightarrow R$ and $h: X \rightarrow R$ are functions satisfying the following assumptions:

(i) $\quad f$ is lower semicontinuous in $y$ on compact subsets of $Y$.

(ii) $e$ is lower semicontinuous on compact subsets of $Y$.

(iii) $f$ is 0 -generalized $G$-H-quasiconcave in $x$.

(iv) There exists an element $x_{0} \in X$ such that the set

$$
\left\{y \in Y: f\left(x_{0}, y\right)+e(y)-h\left(x_{0}\right) \leq 0\right\}
$$

is a compact subset of $Y$.

Then there exists an element $\underset{\sim}{y} \in Y$ such that

$$
f(x, \underset{\sim}{y})+e(\underset{\sim}{y})-h(x) \leq 0 \text { for all } x \in X .
$$

Proof: Let us define a mapping $T: X \rightarrow P(Y)$ by

$$
T(x)=\{y \in Y: f(x, y)+e(y)-h(x) \leq 0\} \text { for all } x \in X \text {. }
$$


Since $f$ is 0 -generalized G-H-quasiconcave, it implies that $T(x)$ is nonempty. It follows from Proposition 2.1 that $T$ is a G-H-KKM mapping. By $(i)$ and (ii), each $T(x)$ is finitely G-H-closed, that is, for each $\underset{\sim}{A} \subset A \in\langle Y\rangle$, we have

$$
\begin{gathered}
T(x)\left(^{-}\right) H(\underset{\sim}{A})=\{y \in H(\underset{\sim}{A}): f(x, y)+e(y)-h(x) \leq 0\} \\
=\{y \in H(\underset{\sim}{A}): f(x, y)+e(y) \leq h(x)\}
\end{gathered}
$$

is closed in $H(\underset{\sim}{A})$ by the lower semicontinuity of $f$ and $e$, so the family $\{T(x): x \in X\}$ has the finite intersection property by Lemma 2.1. Now applying $(i v)$, we find that $\left\{T(x)\left({ }^{-}\right) T\left(x_{0}\right): x \in X\right\}$ is a family of compact subsets of $Y$. Hence, $\left({ }_{x \in X}\right) T(x) \neq \emptyset$. That means, there exists an element $\underset{\sim}{y} \in Y$ such that

$$
f(x, \underset{\sim}{y})+e(\underset{\sim}{y})-h(x) \leq 0 \text { for all } x \in X
$$

This completes the proof.

For $Y$ compact, Theorem 2.1 reduces to:

Theorem 2.2: Let $X$ be a nonempty set and $(Y, H,\{p\})$ a compact $G$-H-space with $H(F)$ compact for all $F \in\langle Y\rangle$. Suppose that $f: X \times Y \rightarrow R, e: Y \rightarrow R$ and $h: X \rightarrow R$ are functions such that:

(i) $\quad f$ is lower semicontinuous in second variable $y$.

(ii) $e$ is lower semicontinuous.

(iii) $f$ is 0 -generalized $G$-H-quasiconcave in first variable $x$.

Then there is an element $\underset{\sim}{y} \in Y$ such that

$$
f(x, \underset{\sim}{y})+e(\underset{\sim}{y})-h(x) \leq 0 \text { for all } x \in X
$$

\section{References}

[1] Chang, S.S. and Zhang, Y., Generalized KKM theorem and variational inequalities, J. Math. Anal. Appl. 159 (1991), 208-223.

[2] Joo', I. and Kassay, G., Convexity, minimax theorems and their applications, Annales Univ. Sci. Budapest 38 (1995), 71-93.

[3] Lin, L.J. and Chang, T.H., S-KKM theorems, saddle points and minimax inequalities, Nonlinear Analysis 34 (1998), 73-86.

[4] Tan, K.K., G-KKM theorem, minimax inequalities and saddle points, Nonlinear Analysis 32 (1997), 4151-4160.

[5] Verma, R.U., Some generalized KKM selection theorems and minimax inequalities, Math. Sci. Res. Hot-Line 2:6 (1998), 13-21.

[6] Verma, R.U., G-H-KKM selections and generalized minimax theorems, Math. Sci. Res. Hot-line 2:8 (1998), 5-10.

[7] Verma, R.U., Role of generalized KKM type selections in a class of minimax inequalities, Appl. Math. Lett. (to appear).

[8] Neumann, J.V. and Morgenstern, O., Theory of Games and Economic Behavior, University Press, Princeton 1944.

[9] Zeidler, E., Nonlinear Functional Analysis and its Applications I, SpringerVerlag, New York 1986. 


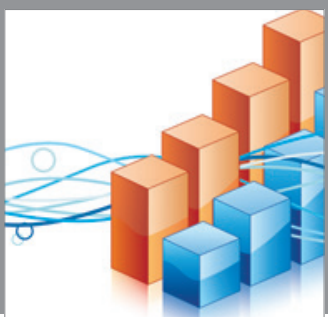

Advances in

Operations Research

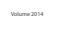

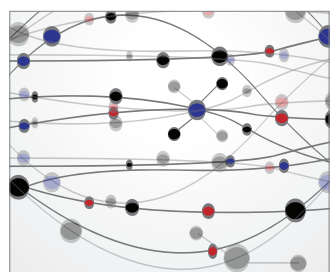

\section{The Scientific} World Journal
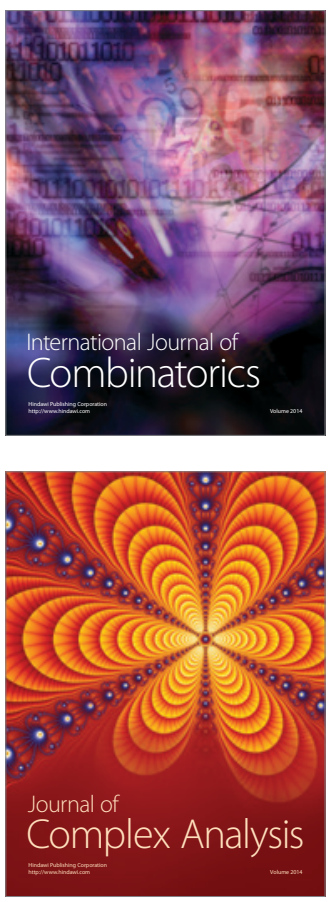

International Journal of

Mathematics and

Mathematical

Sciences
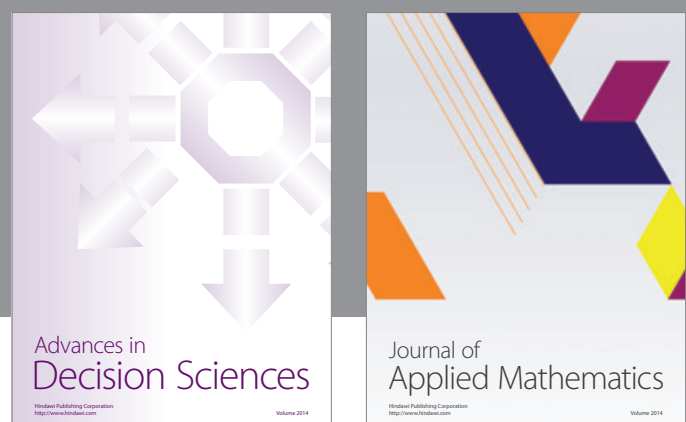

Journal of

Applied Mathematics
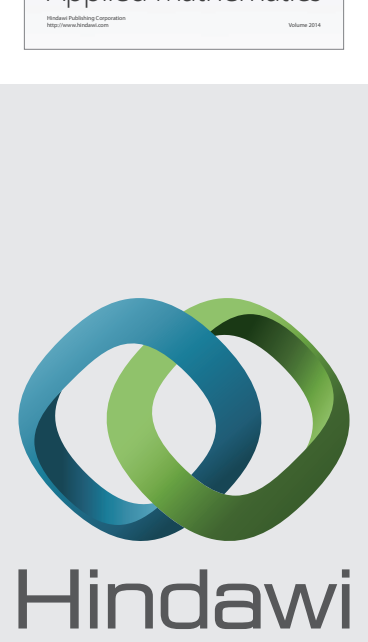

Submit your manuscripts at http://www.hindawi.com
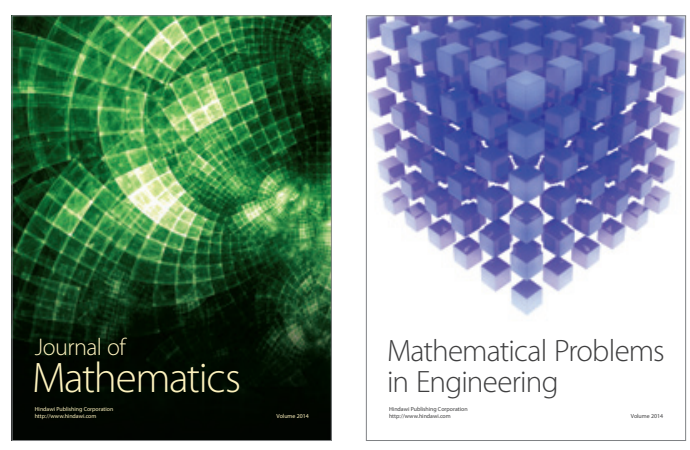

Mathematical Problems in Engineering
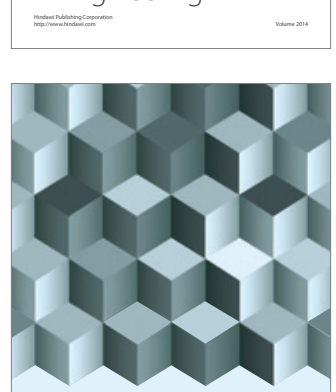

Journal of

Function Spaces
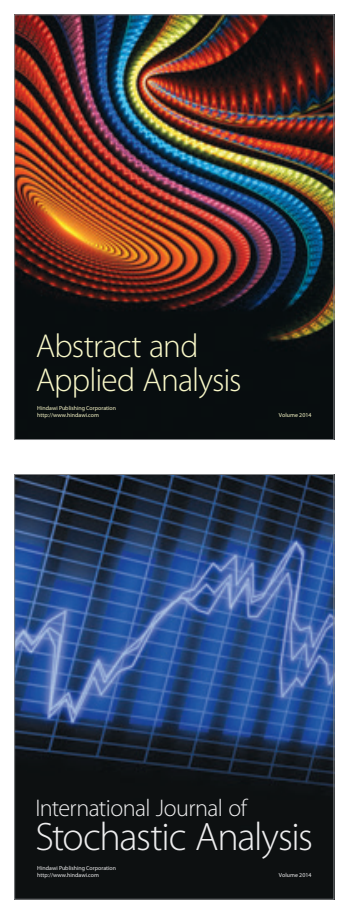

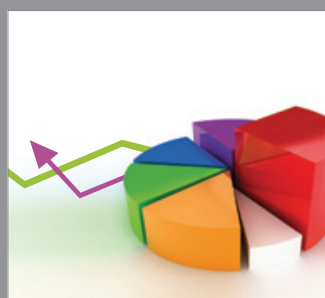

ournal of

Probability and Statistics

Promensencen
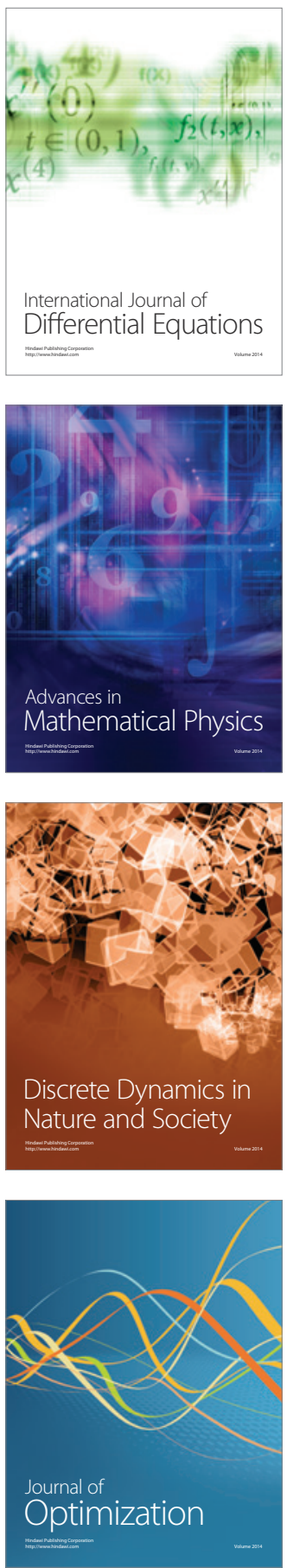